\title{
Outcome of Colorectal Surgery in Elderly Populations
}

\author{
Mostafa Shalaby, Nicola Di Lorenzo, Luana Franceschilli, Federico Perrone, Giulio P. Angelucci, \\ Silvia Quareisma, Achille L. Gaspari, Pierpaolo Sileri \\ Department of General Surgery, UOC B, Policlinico Tor Vergata, University of Rome Tor Vergata, Rome, Italy
}

Purpose: The aim of this study is to investigate the impact of age on short-term outcomes after colorectal surgery in terms of the 30-day postoperative morbidity and mortality rates.

Methods: The subjects for the study were patients who had undergone colorectal surgery. Patients were divided into 2 groups according to age; groups A and B patients were $\geq 80$ and $<80$ years old of age, respectively. Both groups were manually matched for body mass index, American Society of Anesthesiologists score, Charlson Comorbidity Index and procedure performed.

Results: A total of 200 patients, 91 men (45.5\%) and 109 women (54.5\%), were included in this retrospective study. These patients were equally divided into 2 groups. The mean ages were 85 years in group A (range, 80 to 104 years) and 55.3 years in group B (range, 13 to 79 years). The overall 30-day postoperative mortality rate was $1 \%$ of total 200 patients; both of these 2 patients were in group A. However, this observation had no statistical significance. No intraoperative complications were encountered in either group. The overall 30-day postoperative morbidity rate was $27 \%$ (54 of 200) for both groups. The 30-day postoperative morbidity rates in groups A and B were 28\% (28 of 100) and 26\% (26 of 100), respectively. However, these differences between the groups had no statistical significance importance.

Conclusion: Age alone should not be considered to be more of a contraindication or a worse predictor than other factors for the outcome after colorectal surgery on elderly patients.

Keywords: Aged; Colorectal surgery; Colorectal neoplasms; Charlson Comorbidity Index

\section{INTRODUCTION}

According to the World Health Organization (WHO), elderly populations around the world are rapidly growing, with the number of people over 60 years of age expected to reach 2 billion by the year 2050 [1]. Because of the aging of the population, combined with the fact that colorectal surgery is the most commonly performed major surgery on elderly patients $[2,3]$, colorectal surgeons should expect to operate more frequently on elderly patients [4]. Elderly patients are often categorized as high-risk pa-

Received: March 18,2016 Accepted: July 23, 2016

Correspondence to: Mostafa Shalaby, M.D.

Department of General Surgery, UOC B, Policlinico Tor Vergata, University of Rome Tor Vergata, Viale Oxford 81, 00133 Rome, RM, Italy

Tel: +393209353441, Fax: +39620902927

E-mail: mostafashalaby@mans.edu.eg

(C) 2016 The Korean Society of Coloproctology

This is an open-access article distributed under the terms of the Creative Commons Attribution NonCommercial License (http://creativecommons.org/licenses/by-nc/4.0) which permits unrestricted noncommercial use, distribution, and reproduction in any medium, provided the original work is properly cited. tients due to their having significant comorbidity with increasing postoperative morbidity and mortality [5]. The 30-day morbidity and mortality rates are $20 \%$ and $6 \%$, respectively, for patients aged $\geq 70$ years of age $[4,5]$.

Advancing age is considered an important and independent risk factor for postoperative morbidity and mortality; this was confirmed by analysis of the national database in the United States of 975,825 patients aged $\geq 65$ who had undergone a colorectal resection and by a systematic review of 28 independent studies $[4,6]$. The aim of this study was to evaluate the impact of age on shortterm outcomes after colorectal surgery at a single institution.

\section{METHODS}

The subjects for the study were patients who had undergone colorectal surgery for both benign and malignant diseases either as elective or urgent surgery with open or laparoscopic approaches at our department of general surgery. Data from our prospectively collected computer database for dates from June 2003 to September 2010 were extracted, reviewed, and analyzed; further clinical 
information from reviews of clinical notes were also considered. Patients were divided into 2 groups according to age; patients in group A were aged $\geq 80$ years, and those in group B were aged $<80$ years. Both groups were manually matched for BMI, American Society of Anesthesiologists (ASA) score, CCI, and procedure performed (open vs. laparoscopic, elective vs. urgent). All patients signed written informed consent, including consent to possible future publication, according to the Italian bioethics laws. Institutional Review Board approval was obtained from the Ethical Committee of the Policlinico Tor Vergata Hospital in compliance with the Principals of the Helsinki Declaration.

Basic demographic data, including age, sex, BMI, ASA score, disease (benign and malignant), type of surgery (elective or urgent), type of surgical approach (laparoscopic or open), duration of surgery, postoperative hospital stay, postoperative morbidity, and postoperative mortality were recorded for each patient. All patients were evaluated preoperatively by expert anesthesiologists for individual comorbidities with quantification using the weighted CCI and classification according to the ASA score. The CCI is a method with 22 variables, which was described by Charlson et al. [7], for measuring comorbidity [6, 7]. Antibiotic prophylaxis was given to all patients at the induction of anesthesia in the form of $1 \mathrm{gm}$ of cephalosporin, with a second dose being administered if the surgery exceeded four hours. Adequate thromboembolic prophylaxis was given in the form of low-molecularweight heparin (4,000 IU/day) 8 hours before an elective procedure or 8 hours after an urgent procedure. All operations were performed by experienced surgeons.

Analysis was performed with SPSS ver. 12.0 (SPSS Inc., Chicago, IL, USA). Categorical variables were analyzed with the chi-square test, Fisher exact test or the Student t-test for quantitative and qualitative variables, as appropriate. Data were expressed as median and range unless specified otherwise. $\mathrm{P} \leq 0.05$ was considered to be statistically significant.

\section{RESULTS}

A total number of 200 patients, 91 men (45.5\%) and 109 women (54.5\%), were included in this retrospective study. These patients

Table 1. Patients' demographic parameters, ASA scores, and CCIs

\begin{tabular}{lcc}
\hline Variable & Group A & Group B \\
\hline No. of patients & 100 & 100 \\
Mean age $(\mathrm{yr})$ & 85 & 55.3 \\
Sex, male : female & $37: 63$ & $54: 46$ \\
Body mass index $\geq 30 \mathrm{~kg} / \mathrm{m}^{2}$ & $4 \%$ & $5 \%$ \\
ASA Score $\geq 3$ & $24 \%$ & $16 \%$ \\
Mean CCl score & 3.1 & 2.8 \\
\hline
\end{tabular}

Group $A, \geq 80$ years old of age; group $B,<80$ years old of age.

ASA, American Society of Anesthesiologist; CCl, Charlson Comorbidity Index. were equally divided into two groups: groups $\mathrm{A}$ and $\mathrm{B}$ patients were $\geq 80$ and $<80$ years old of age, respectively. The mean ages were 85 years in group A (range, 80 to 104 years), and 55.3 years in group $\mathrm{B}$ (range, 13 to 79 years). The basic demographic parameters of both groups, including BMI, ASA scores, and mean CCI, are listed in Table 1 . No statistically significant differences were noted between the 2 groups regarding BMI, ASA, CCI, type of intervention (elective vs. urgent), and surgical approaches (open vs. laparoscopic) (Table 2). These results were to be expected as the 2 groups were manually matched for these items. The causes of admission to the hospital (Table 2) were malignant diseases in 56\% and $58 \%$ of the patients in groups A and B, respectively, with comparable TNM stages for both groups.

The mean total operative times were 136 minutes (range, 40 to 255 minutes) in group A and 155 minutes (range, 30 to 355 minutes) in group B (Table 3). In particular, the mean operative times for the laparoscopic procedures were 122.5 minutes (range, 45 to 230 minutes) in group A and 146.8 minutes (range, 40 to 315 minutes) in group $\mathrm{B}$ while those for the open procedures were 138 minutes (range, 35 to 255 minutes) in group A and 158.9 minutes (range, 30 to 355 minutes) in group $\mathrm{B}$. The mean lengths of hospital stay for group A (range, 5 to 41 days) and group B (range, 4 to 31 days) were statistically the same (13 days, Table 3 ).

The overall 30 -day postoperative mortality rate was $1 \%$ (2 of 200) for the total of both groups, with group A having both fatalities and group A having none; however, this difference was not

Table 2. Disease type, type of intervention, and surgical procedure

\begin{tabular}{lcc}
\hline & Group A & Group B \\
\hline Malignant & $56 \%$ & $58 \%$ \\
Elective & $63 \%$ & $52 \%$ \\
Urgent & $37 \%$ & $48 \%$ \\
Obstruction & $11 \%$ & $17 \%$ \\
Perforation & $8 \%$ & $4 \%$ \\
Laparoscopic & $17 \%$ & $27 \%$ \\
Traditional & $83 \%$ & $73 \%$ \\
\hline
\end{tabular}

Group $A, \geq 80$ years old of age; group $B,<80$ years old of age.

Table 3. Operative and procedure times, hospital stays, mortalities and morbidities for the 2 groups

\begin{tabular}{lcc}
\hline & Group A & Group B \\
\hline Mean total operation time (min) & 136 & 155 \\
Mean time in laparoscopic procedures (min) & 122.5 & 146.8 \\
Mean time in open procedures (min) & 138 & 158.9 \\
Mean hospital stay (day) & 13 & 13 \\
Mortality (\%) & 2 & 0 \\
Morbidity (\%) & 28 & 26 \\
\hline
\end{tabular}

Group A, $\geq 80$ years old of age; group B, $<80$ years old of age. 
statistically significant (Table 3 ). One of those 2 patients was 81 years old, ASA grade 2, who had undergone an anterior resection for rectal cancer, followed by a Hartmann procedure for peritonitis due to anastomotic leakage; that patient died 14 days after the initial procedure. The second was 86 years old, ASA grade 3, who had undergone a Miles procedure for rectal cancer and then developed acute coronary artery syndrome with acute heart failure; that patient died 7 days after the initial procedure.

No intraoperative complications were noted in either group. The overall 30-day postoperative morbidity rate was $27 \%$ (54 of 200) for the total of both groups. The 30-day postoperative morbidity rate in group A was $28 \%$ (28 of 100). Of these 28 patients, $18 \%$ (5 of 28 ) had CCI $\geq 3$, and $36 \%$ (10 of 28 ) were classified ASA $\geq$ III. The 30 -day postoperative morbidity rate was $26 \%$ (26 of 100) for group B. Of these 26 patients, $7.6 \%$ ( 2 of 26) had CCI $\geq 3$, and $23 \%$ ( 6 of 26 ) were classified ASA $\geq 3$. However, these differences between the 2 groups showed no statistical significance (Table 3). Of the patients developing complication (54 total, 28 in group A and 26 in group B), those requiring addition surgical interventions were 9 total ( 9 of 54, 17\%), 4 ( 4 of 28, 14\%) in group A, and 5 (5 of $26,19 \%)$ in group B (Table 4$)$. The additional operative interventions were a Hartmann procedure for anastomotic leakage $(\mathrm{n}=1$, group $\mathrm{A})$ and protective ileostomies for anastomotic leakage ( $\mathrm{n}=2,1$ in each group).

\section{DISCUSSION}

One of the challenges in evaluating the outcomes of colorectal surgery in elderly patients is that no consistent definition of an elderly patient exists, with ages varying from 65 to 80 years in different publications [5]. Other challenges are the varying definitions of morbidity in different institutions and the fact that several complications, like postoperative delirium, may go unrecognized and be unreported [3]. Elderly is a very subjective term based mainly on the environmental culture of the patient. Elderly has been defined as a chronological age of $\geq 65$ years of age, which has been further subdivided into early and late elderly for those who are 65 through 74 and over 75 years of age, respectively [8]. To the best of our knowledge, this is the first study about the outcome of

Table 4. Patients requiring additional surgery

\begin{tabular}{lll}
\hline & Group A & Group B \\
\hline No of patients (\%) & $4(14)$ & $5(19)$ \\
Anastomotic leakage & 2 & 1 \\
Anastomotic bleeding & 1 & - \\
Intra-abdominal bleeding & - & 1 \\
Small bowel obstruction & 1 & 1 \\
Intra-abdominal abscess & - & 1 \\
lleostomy necrosis & - & 1 \\
\hline
\end{tabular}

Group $A, \geq 80$ years old of age; group $B,<80$ years old of age. colorectal surgery in elderly patients with comparison to a cohort group of nonelderly patients manually matched for BMI, ASA score, CCI and procedure performed (open vs. laparoscopic, elective vs. urgent).

In this study, we set the cut off age defining elderly at 80 years of age as it is widely used [9]. Comorbidity was weighted using the CCI, in addition to the ASA score. The ASA score is a known prognostic factor for increased morbidity and mortality; elderly patients often have a ASA score $\geq 2$ [10]. The use of the CCI could ensure more reliable and objective decision-making as it represents a good, easily calculated, and feasible tool for evaluating comorbid conditions of patients and for predicting postoperative morbidity and 10-year survivals. The surgical risk is directly correlated to the number of comorbidities of a patient, with a patient having a $\mathrm{CCI}>3$ having a 1 -year mortality double that of a patient having a CCI $<3$ [9]. However, the assigned weights have limitations: "Any malignancy" was "any tumor" in the original table without differentiation between benign or malignant tumors, and good or poor prognosis malignancies. In addition, the survival of patients with acquired immunodeficiency syndrome has improved since 1987 [9]. Another limitation is that the CCI is somewhat still a geriatrician's tool [11].

No statistically significant differences were noted between the 2 groups regarding BMI, ASA score, CCI, type of intervention (elective vs. urgent), and surgical approach (open vs. laparoscopic) (Tables 1, 2). These results were expected as both groups were manually matched for these items. No statistically significant differences were observed in term of operative time between the 2 groups for patients with postoperative complications. In particular, the $54 \%$ and the $30 \%$ of the patients with postoperative complications in groups $\mathrm{A}$ and $\mathrm{B}$, respectively, required surgical procedures with durations $\geq 180$ minutes. The mean lengths of hospital stay for group A (range, 5 to 41 days) and group B (range, 4 to 31 days) were statistically the same (13 days) (Table 3 ). This result concurs with a published result [8].

No intraoperative complications were encountered in either group. The overall 30-day postoperative mortality rate was $1 \%$ (2 of 200) for the total number of patients, with group A accounting for both fatalities, and group B for none. The overall 30-day postoperative morbidity rate was $27 \%$ (54 of 200) for the total number of patients, $28 \%$ (28 of 100) for group A, and 26\% (26 of 100) for group B. However, these differences between the 2 groups were not statistically significant (Table 3).

Statistically significant results in postoperative morbidity were observed between the 2 groups for the duration of the procedures and the type of the procedure elective $(\mathrm{P}=0.0005)$ or urgent $(\mathrm{P}=$ 0.0003). In group A, 28 patients developed postoperative complications; 8 (28.6\%) were subjected to urgent procedures, and 20 (71.4\%) were subjected to elective procedures. On the other hand, in group $\mathrm{B}$, the numbers of elective and urgent procedures were similar. Of the 26 patients who developed postoperative complications in group B, 12 (46\%) were subjected to urgent procedures, 
and 14 (54\%) were subjected to elective procedures. These results are similar to those published elsewhere [11-14]. This explain that emergency surgery patients usually have compromised general conditions and there no more time to correct [14]. Surgeons and physicians should encourage elderly patients to undergo elective surgery to manage the complication of their diseases rather than wait and ultimately being required to undergo emergent surgery. The reported mortality rate for emergency procedures varies between $6.1 \%-38 \%$ for patients older than 70 years of age and between $11.9 \%-38 \%$ for those older than 80 years of age, respectively, compared to $0.9 \%-18 \%$ and $7.4 \%-11.4 \%$ for the same age groups for elective procedures [15]. In a trail to address the morbidity and the mortality in elderly patients who underwent emergency colorectal surgery, McGillicuddy et al. [14] studied 292 patients with a mean age of 78.1 years. They found an increase in the mean hospital stay of 20.9 days, an in-hospital mortality of $15 \%$, and morbidities of $67 \%$.

Our results did not report statistically significant differences between elderly and nonelderly patients regarding postoperative morbidity and mortality, concur with recent studies published. A review article by Tan et al. [3] stated that elderly patients had a higher complications rate, but still had a low mortality, especially for emergent procedures. A study by Schwandner et al. [2] included 298 patients who had undergone laparoscopic or laparoscopic-assisted procedures for colorectal surgery. The morbidity in patients above 70 years of age and that in patients below 70 years of age showed no statistically significant difference. Also, 2 patients above 70 years of age died versus and 1 patient below that age. Tan et al. [11] studied 121 patients with a mean age of 83.5 years and a mean CCI of 3.1 who had undergone major colorectal resections; the 30 -day mortality was $1.6 \%$, and the early complications rate was $30.6 \%$.

Ong et al. [12] included 90 patients 80 years of age or older who had undergone colorectal cancer surgery; they reported a morbidity of $21 \%$ and a 30 -day mortality of $1.1 \%$. Basili et al. [13] reported their experience with 248 patients who had undergone colorectal cancer surgery. Patients were divided into 4 age groups: $<65,66$ to 74,75 to 84 , and $>85$ years of age. The 30 -day mortalities were $0 \%$ for under 75 years of age, and $6 \%$, and $7 \%$, respectively, for patients with ages from 75 to 84 years and those older than 85 years. However, none of these results was significant.

In a large multicenter prospective observational study in Germany on 16,142 patients who were younger than 80 years of age and 2,932 who were 80 years of age or older, Marusch et al. [15] reported an overall morbidity of $35.4 \%$ with a significant difference $(\mathrm{P}<0.001)$ between patients $<80$ years of age $(33.9 \%)$ and those $\geq 80$ years of age (43.5\%). Significant differences were also found between the morbidity for emergency surgery $(\mathrm{P}<0.001)$ and that for elective surgery $(\mathrm{P}<0.001)$. The 30 -day postoperative mortality rate also differed significantly $(\mathrm{P}<0.001) ; 2.1 \%$ and $7.2 \%$ for those $<80$ years of age and those $\geq 80$ years of age, respectively. Despite these significant results, they concluded that age alone should not be a limitation for surgery.

Despite these efforts, our study still has certain limitations that must be discussed. First, this study was retrospective. Second, the number of patients in the current study was not very high. Third, the CCI as a method for evaluating comorbidity and for predicting postoperative complications has the previously mentioned limitations. In the future, multicenter, randomized, controlled trials of a prospective nature comparing the outcomes of colorectal surgery in elderly patients to those in nonelderly patients should be conducted. Advanced age alone should not be considered to be more of a contraindication or a worse predictor for the outcome after colorectal surgery. Pre-existing comorbidity and the urgent nature of the procedure are important predictors for the outcome after colorectal surgery in elderly patients.

\section{CONFLICT OF INTEREST}

No potential conflict of interest relevant to this article was reported.

\section{REFERENCES}

1. World Health Organization. Ageing and life-course [Internet]. Geneva: World Health Organization; c2016 [cited 2015 Aug 6]. Available from: http://www.who.int/ageing/en/.

2. Schwandner O, Schiedeck TH, Bruch HP. Advanced age: indication or contraindication for laparoscopic colorectal surgery? Dis Colon Rectum 1999;42:356-62.

3. Tan KY, Konishi F, Tan L, Chin WK, Ong HY, Tan P. Optimizing the management of elderly colorectal surgery patients. Surg Today 2010;40:999-1010.

4. Tan WS, Chew MH, Lim IA, Ng KH, Tang CL, Eu KW. Evaluation of laparoscopic versus open colorectal surgery in elderly patients more than 70 years old: an evaluation of 727 patients. Int J Colorectal Dis 2012;27:773-80.

5. Seishima R, Okabayashi K, Hasegawa H, Tsuruta M, Shigeta K, Matsui S, et al. Is laparoscopic colorectal surgery beneficial for elderly patients? A systematic review and meta-analysis. J Gastrointest Surg 2015;19:756-65.

6. Miyasaka Y, Mochidome N, Kobayashi K, Ryu S, Akashi Y, Miyoshi A. Efficacy of laparoscopic resection in elderly patients with colorectal cancer. Surg Today 2014;44:1834-40.

7. Charlson ME, Pompei P, Ales KL, MacKenzie CR. A new method of classifying prognostic comorbidity in longitudinal studies: development and validation. J Chronic Dis 1987;40:373-83.

8. Bircan HY, Koc B, Ozcelik U, Adas G, Karahan S, Demirag A. Are there any differences between age groups regarding colorectal surgery in elderly patients? BMC Surg 2014;14:44.

9. Roscio F, Bertoglio C, De Luca A, Frigerio A, Galli F, Scandroglio I. Outcomes of laparoscopic surgery for colorectal cancer in elderly patients. JSLS 2011;15:315-21.

10. Feng B, Zheng MH, Mao ZH, Li JW, Lu AG, Wang ML, et al. 
Clinical advantages of laparoscopic colorectal cancer surgery in the elderly. Aging Clin Exp Res 2006;18:191-5.

11. Tan KY, Kawamura Y, Mizokami K, Sasaki J, Tsujinaka S, Maeda T, et al. Colorectal surgery in octogenarian patients--outcomes and predictors of morbidity. Int J Colorectal Dis 2009;24:185-9.

12. Ong ES, Alassas M, Dunn KB, Rajput A. Colorectal cancer surgery in the elderly: acceptable morbidity? Am J Surg 2008;195: 344-8.

13. Basili G, Lorenzetti L, Biondi G, Preziuso E, Angrisano C, Carne- secchi $\mathrm{P}$, et al. Colorectal cancer in the elderly. Is there a role for safe and curative surgery? ANZ J Surg 2008;78:466-70.

14. McGillicuddy EA, Schuster KM, Davis KA, Longo WE. Factors predicting morbidity and mortality in emergency colorectal procedures in elderly patients. Arch Surg 2009;144:1157-62.

15. Marusch F, Koch A, Schmidt U, Steinert R, Ueberrueck T, Bittner R, et al. The impact of the risk factor "age" on the early postoperative results of surgery for colorectal carcinoma and its significance for perioperative management. World J Surg 2005;29:1013-21. 